\title{
Reciprocating and Directed Motion on the Nanoscale: A Simple Kinetic Model
}

\author{
V. M. Rozenbaum, ${ }^{* \dagger, \sharp}$ Yu. A. Makhnovskii, ${ }^{\dagger,}$ D.-Y. Yang, ${ }^{\dagger}$ S.-Y. Sheu, and S. H. Lin ${ }^{\dagger, \perp}$ \\ Institute of Atomic and Molecular Sciences, Academia Sinica, Taipei 106, Taiwan; Chuiko Institute of Surface \\ Chemistry, National Academy of Sciences of Ukraine, Generala Naumova str. 17, Kiev, 03164, Ukraine; Topchiev \\ Institute of Petrochemical Synthesis, Russian Academy of Sciences, Leninsky Prospect 29, 119991 Moscow, Russia; \\ Department of Life Sciences and Institute of Genome Sciences, Institute of Biomedical Informatics, and Structural \\ Biology Program, National Yang-Ming University, Taipei 112, Taiwan; and Department of Applied Chemistry, \\ National Chiao Tung University, 1001 Ta Hsuen Road, Hsinchu, Taiwan
}

Received: November 3, 2009

\begin{abstract}
We consider noise-induced reciprocating motion on the nanoscale and its rectification to directed motion using a simple model in which transitions between two fluctuating states occur through two reaction channels with fluctuating transition rates. The fluctuations of states and transition rates arise from equilibrium thermal and external nonthermal noise which is in either case position-dependent. The model is equivalent to a Brownian particle hopping in a periodic double-well potential which randomly switches between two profiles. With a nonequilibrium noise, a generalized driving force may be regarded as the sum of two forces: one resulting from energy fluctuations and the other from fluctuations of the spatial dependence of the transition rates. This suggests two mechanisms, energetic and informational, by which the motion occurs. The reciprocating motion results in directed motion if rectified by asymmetric fluctuations of potential barriers. The energy conversion efficiency is calculated and the conditions to maximize it are established.
\end{abstract}

\section{Introduction}

Unbiased nonequilibrium fluctuations produced by a chemical reaction or any other external process can cause directed motion in small-scale systems. The problem of noise-induced transport has been discussed in different contexts by approaches of varying rigor and sophistication. ${ }^{1-6}$ Investigations in this area are primarily aimed at gaining insight into the operation principles of molecular motor proteins ${ }^{7}$ and ion pumps. ${ }^{8}$ Another motivation comes from artificial molecular ${ }^{9}$ and nanoscale machinery design: ${ }^{10}$ this rapidly developing field calls for synthetic structures which, if supplied with energy and information, can controllably move molecular-level systems and interface them with the macroscopic world. ${ }^{6}$

Unbiased nonequilibrium fluctuations in an intrinsically structured and conformationally flexible system can induce oppositely directed motions in different conformations. If the system is symmetric, such motions are mutually canceled so that no net motion occurs. The input energy, though converted to the mechanical energy of undirected motion on the nano length/time scale, is finally dissipated as heat. In what follows, motion of this kind (resembling a futile circle in biochemistry ${ }^{11}$ ) will be referred to as reciprocating motion. Provided a symmetry-breaking rectification mechanism is present, the reciprocating motion can be partially converted to directed motion. With this strategy, energy conversion involves two steps, by analogy with a macroscopic combustion engine: the energy supplied by a nonequilibrium source is first converted to reciprocating mechanical motion at the nanolevel, which is then rectified.

\footnotetext{
* Corresponding author. E-mail: vrozen@isc.gov.ua.

† Academia Sinica.

* National Academy of Sciences of Ukraine.

$\S$ Russian Academy of Sciences.

"National Yang-Ming University.

${ }^{\perp}$ National Chiao Tung University.
}

The present paper addresses the theory of the abovementioned two-step energy conversion. Unlike previous studies ${ }^{12,13}$ invoking the continuous Fokker-Planck description, here we attack the problem by using the discrete chemical kinetic method $^{5,14-20}$ which is analytically easier and often provides exact solutions for complex stochastic models in terms of transition rates between the accessible states of the system. We refine the previously developed model ${ }^{21}$ by incorporating the position dependence of the interconformation transition rate constants. With this generalization, the mode ${ }^{22}$ includes the spatial dependence of the system reactivity that fundamentally improves its biological relevance. We show that the reciprocating motion is caused not only by well and barrier fluctuations but also by fluctuations of the spatial dependence of the transition rates. The energetic and the entropic contributions to the driving force suggest two mechanisms of the motion generation: energetic and informational. We also show that the directed motion arises due to a rectifying effect of asymmetric fluctuations of potential barriers.

The general kinetic analysis of the model is presented in section II. The derived expressions for the net circulation and reciprocating fluxes are discussed in terms of an equivalent flashing potential model in section III. For the antisymmetric version of the model (see section IV), explicit formulas are obtained for both the fluxes, the rectification coefficient, and the energy conversion efficiency. This allows us to reveal some interesting regularities, as for instance, the conditions for highefficiency operation of the Brownian motor under study.

\section{General Kinetic Model}

Consider a system with two spatially separated states A and $\mathrm{B}$, each having two randomly interchanging conformations "+" and "-". Assume that intraconformational transitions occur through two reaction channels $\alpha$ and $\beta$, with rate constants $\alpha(\beta)_{\mathrm{AB}}^{ \pm}$and $\alpha(\beta)_{\mathrm{BA}}^{ \pm}$(see Figure 1a), and are caused by equilib- 


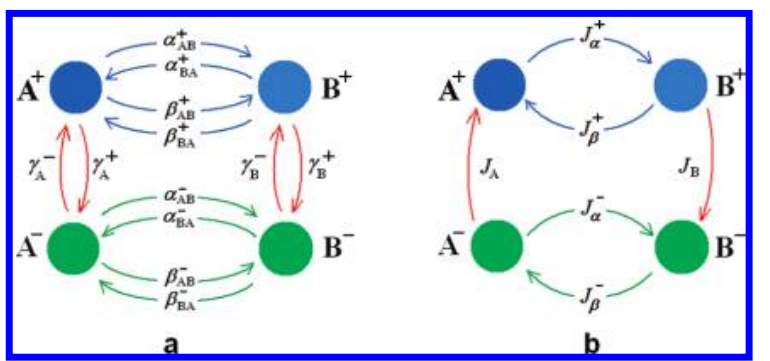

Figure 1. Schematic representation of a kinetic model in which transitions between two states A and B, each having two randomly interchanging conformations + and - , occur through two reaction channels with fluctuating transition rates $\alpha^{ \pm}$and $\beta^{ \pm}$. The interconformational transitions are characterized by rate constants $\gamma_{\mathrm{A}, \mathrm{B}}^{ \pm}$, each specified by the corresponding state and conformation. Panels (a) and (b) show diagrams for the rate constants and the corresponding fluxes, respectively.

rium (thermal) fluctuations only. Assume also that interconformational jumps are due to both thermal and nonthermal (external) noise and characterized by certain rate constants $\gamma_{\mathrm{A}, \mathrm{B}}^{ \pm}$, each specified by the corresponding state and conformation. Let $\rho_{\mathrm{A}}^{ \pm}$and $\rho_{\mathrm{B}}^{ \pm}$denote the respective probabilities for the system to be in states $\mathrm{A}$ and $\mathrm{B}$, with regard to the conformations involved.

The reciprocating motion between $\mathrm{A}$ and $\mathrm{B}$ can easily be conceived if the system has the only reaction channel $\alpha$. Then the probability flux through channel $\alpha$ between states A and B as well as the fluxes between the + and - conformations within $\mathrm{A}$ and $\mathrm{B}$ appear as (see Figure 1 neglecting channel $\beta$ ):

$$
\begin{aligned}
J_{\alpha}^{ \pm}=\alpha_{\mathrm{AB}}^{ \pm} \rho_{\mathrm{A}}^{ \pm}-\alpha_{\mathrm{BA}}^{ \pm} \rho_{\mathrm{B}}^{ \pm}, \quad J_{\mathrm{B}}=\gamma_{\mathrm{B}}^{+} \rho_{\mathrm{B}}^{+}-\gamma_{\mathrm{B}}^{-} \rho_{\mathrm{B}}^{-}, \\
J_{\mathrm{A}}=\gamma_{\mathrm{A}}^{-} \rho_{\mathrm{A}}^{-}-\gamma_{\mathrm{A}}^{+} \rho_{\mathrm{A}}^{+}
\end{aligned}
$$

In a steady-state regime, state populations do not vary with time $\left(\mathrm{d} \rho_{\mathrm{A}}^{ \pm} / \mathrm{d} t=\mathrm{d} \rho_{\mathrm{B}}^{ \pm} / \mathrm{d} t=0\right)$, so that $J_{\mathrm{A}}=J_{\alpha}^{+}=-J_{\alpha}^{-}=J_{\mathrm{B}}$. It is natural to define the reciprocating flux between states $\mathrm{A}$ and $\mathrm{B}$ as $J_{\mathrm{r}}=$ $J_{\mathrm{A}}=J_{\mathrm{B}}$. The net circulation flux $J=J_{\alpha}^{+}+J_{\alpha}^{-}$is nullified thus ruling out directed circulation.

The second reaction channel $\beta$, with the corresponding transition rate constants $\beta_{\mathrm{AB}}^{ \pm}$and $\beta_{\mathrm{BA}}^{ \pm}$(see Figure 1a), gives rise to an additional flux $J_{\beta}^{ \pm}=\beta_{\mathrm{BA}}^{ \pm} \rho_{\mathrm{B}}^{ \pm}-\beta_{\mathrm{AB}}^{ \pm} \rho_{\mathrm{A}}^{ \pm}$. Then the steadystate relations between the fluxes read $J_{\mathrm{A}}=J_{\alpha}^{+}-J_{\beta}^{+}=J_{\mathrm{B}}$ and $J_{\mathrm{B}}=J_{\beta}^{-}-J_{\alpha}^{-}=J_{\mathrm{A}}$ (see Figure $1 \mathrm{~b}$ ). The reciprocating flux is found as before, whereas the net circulation flux is now given by $J=J_{\alpha}^{+}+J_{\alpha}^{-}=J_{\beta}^{+}+J_{\beta}^{-}$. The latter suggests that two reaction channels permit the emergence of directed circular motion under nonequilibrium conditions, ${ }^{21}$ which is especially evident when one conformation has the only reaction channel $\alpha$ and the other the only alternative channel $\beta$ (e.g., $J_{\beta}^{+}=0$ and $J_{\alpha}^{-}=0$ ). In this case, the reverse fluxes are blocked and reciprocating motion is completely rectified. Generally, when motion is induced by unbiased nonequilibrium noise, it is reasonable to introduce the rectification coefficient $\kappa$ defined as the ratio of circulation to reciprocating flux:

$$
\kappa \equiv J / J_{\mathrm{r}}
$$

The coefficient $\kappa$ characterizes the degree of conversion from reciprocating to directed motion. It should be emphasized that such a definition (and, moreover, our two-step approach to energy conversion, which implies $\kappa \leq 1$ ) becomes meaningless if the fluxes $J$ and $J_{\mathrm{r}}$ have different origins (an example of such situation is presented below).

Expressions for the quantities of interest in terms of the transition rate constants are derived from the stationary solution of the master equation for the probabilities $\rho_{\mathrm{A}(\mathrm{B})}^{ \pm}$(see Appendix 1):

$$
\begin{gathered}
J=\Delta^{-1} \sum_{\sigma^{\prime} \neq \sigma= \pm}\left[( \alpha _ { \mathrm { AB } } ^ { \sigma ^ { \prime } } \beta _ { \mathrm { BA } } ^ { \sigma ^ { \prime } } - \alpha _ { \mathrm { BA } } ^ { \sigma ^ { \prime } } \beta _ { \mathrm { AB } } ^ { \sigma ^ { \prime } } ) \left(\xi_{\mathrm{B}}^{\sigma} \gamma_{\mathrm{A}}^{\sigma}+\xi_{\mathrm{A}}^{\sigma} \gamma_{\mathrm{B}}^{\sigma}+\right.\right. \\
\left.\left.\gamma_{\mathrm{A}}^{\sigma} \gamma_{\mathrm{B}}^{\sigma}\right)+\left(\alpha_{\mathrm{AB}}^{\sigma} \beta_{\mathrm{BA}}^{\sigma^{\prime}}-\alpha_{\mathrm{BA}}^{\sigma^{\prime}} \beta_{\mathrm{AB}}^{\sigma}\right) \gamma_{\mathrm{A}}^{\sigma^{\prime}} \gamma_{\mathrm{B}}^{\sigma}\right] \\
J_{r}=\Delta^{-1}\left(\xi_{\mathrm{A}}^{+} \xi_{\mathrm{B}}^{-} \gamma_{\mathrm{B}}^{+} \gamma_{\mathrm{A}}^{-}-\xi_{\mathrm{B}}^{+} \xi_{\mathrm{A}}^{-} \gamma_{\mathrm{A}}^{+} \gamma_{\mathrm{B}}^{-}\right)
\end{gathered}
$$

where $\Delta$ is defined by formula (A1.5) and the following notation is used:

$$
\xi_{\mathrm{A}}^{ \pm}=\alpha_{\mathrm{AB}}^{ \pm}+\beta_{\mathrm{AB}}^{ \pm}, \quad \xi_{\mathrm{B}}^{ \pm}=\alpha_{\mathrm{BA}}^{ \pm}+\beta_{\mathrm{BA}}^{ \pm}
$$

Note that the expression for the net circulation flux, eq 3 , in fact coincides with the solution for the mean velocity of a particle moving along two periodic parallel coupled chains with four different cites in each period obtained in ref 18 (see eq 11 there), where the same kinetic model was considered to study its dynamical properties, such as mean velocity and diffusion coefficient.

It is readily seen from eqs 3 and 4 that both $J$ and $J_{\mathrm{r}}$ vanish if the detailed balance conditions are satisfied (the transitions are driven by thermal noise only):

$$
\alpha_{\mathrm{AB}}^{ \pm} \beta_{\mathrm{BA}}^{ \pm}-\alpha_{\mathrm{BA}}^{ \pm} \beta_{\mathrm{AB}}^{ \pm}=0, \quad \xi_{\mathrm{A}}^{+} \xi_{\mathrm{B}}^{-} \gamma_{\mathrm{B}}^{+} \gamma_{\mathrm{A}}^{-}-\xi_{\mathrm{B}}^{+} \xi_{\mathrm{A}}^{-} \gamma_{\mathrm{A}}^{+} \gamma_{\mathrm{B}}^{-}=0
$$

Condition 1 specifies equilibrium within either conformation and condition 2 between the conformations. When imposed together, the two conditions imply a fully equilibrium state of the system under study.

Note that if the rate constants of the $\alpha$ and $\beta$ channels are the same for both conformations, then fluxes 3 and 4 take the form

$$
\begin{gathered}
J=\frac{\alpha_{\mathrm{AB}} \beta_{\mathrm{BA}}-\alpha_{\mathrm{BA}} \beta_{\mathrm{AB}}}{\xi_{\mathrm{A}}+\xi_{\mathrm{B}}} \\
J_{\mathrm{r}}=\frac{\xi_{\mathrm{A}} \xi_{\mathrm{B}}\left(\gamma_{\mathrm{B}}^{+} \gamma_{\mathrm{A}}^{-}-\gamma_{\mathrm{A}}^{+} \gamma_{\mathrm{B}}^{-}\right)}{\left(\xi_{\mathrm{A}}+\xi_{\mathrm{B}}\right)\left[\xi_{\mathrm{B}}\left(\gamma_{\mathrm{A}}^{+}+\gamma_{\mathrm{A}}^{-}\right)+\xi_{\mathrm{A}}\left(\gamma_{\mathrm{B}}^{+}+\gamma_{\mathrm{B}}^{-}\right)+\right.} \\
\left.\left(\gamma_{\mathrm{A}}^{+}+\gamma_{\mathrm{A}}^{-}\right)\left(\gamma_{\mathrm{B}}^{+}+\gamma_{\mathrm{B}}^{-}\right)\right]
\end{gathered}
$$

In this case, it follows from eq 7 that directed motion (i) does not depend on $\gamma_{\mathrm{A}}^{ \pm}$and $\gamma_{\mathrm{B}}^{ \pm}$; (ii) can occur only in a nonequilibrium state within either conformation (caused, e.g., by a generalized external force); and (iii) requires the existence of two reaction channels. On the other hand, eq 8 shows that reciprocating motion (i) can occur only in the absence of interconformational equilibrium, i.e., at such state-dependent rate constants $\gamma_{\mathrm{A}}^{ \pm}$and $\gamma_{\mathrm{B}}^{ \pm}$that $\gamma_{\mathrm{A}}^{ \pm} \neq \gamma_{\mathrm{B}}^{ \pm}$and $\gamma_{\mathrm{B}}^{+} \gamma_{\mathrm{A}}^{-}-\gamma_{\mathrm{A}}^{+} \gamma_{\mathrm{B}}^{-} \neq 0$; and (ii) requires the only reaction channel $\left(\alpha\right.$ or $\beta$ ). Note that the fluxes $J$ and $J_{\mathrm{r}}$ have different origins. So the case of nonfluctuating rate constants $\alpha$ 's and $\beta$ 's is an example where the two-step approach to energy conversion is inapplicable (see remark after eq 2).

In what follows, we exploit the results of this section to treat not only the fluxes but also energy conversion efficiency (the ratio of mechanical energy output to energy input). We then proceed to determine explicit expressions for the $\alpha$ - and $\beta$-channel rate constants in terms of conformation potential profiles.

\section{Flashing Potential Model}

The above-presented general kinetic model can be well illustrated by an equivalent model of Brownian particle motion 


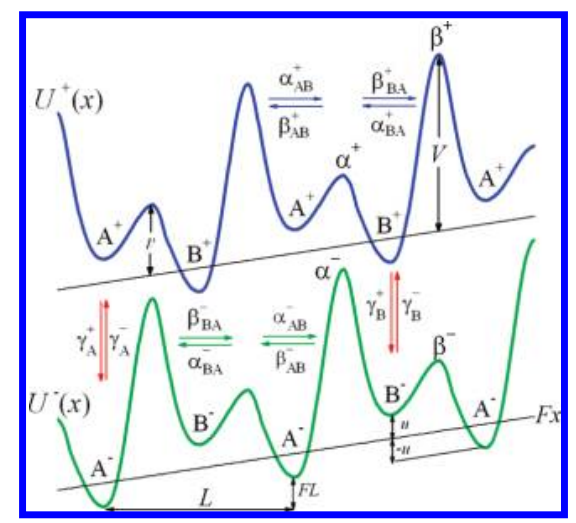

Figure 2. Juxtaposition of the flashing potential model to the simple kinetic model presented in Figure 1.

in a fluctuating periodic two-well potential. With the kinetic approach, the particle can be found in either conformationdependent potential well corresponding to state A or B, and it can surmount interwell barriers, with two conformation-dependent heights, just as the reaction in the kinetic model can proceed through two reaction channels $\alpha$ and $\beta$ (see Figure 2). The potential minima and maxima fluctuate between two value sets, by analogy with the fluctuations of the states A and B and the $\alpha$ - and $\beta$-channel rate constants. The principal difference between the present model and the models of this type proposed earlier ${ }^{15,21,23}$ is that here not only the intraconformational but also the interconformational rate constants are position dependent, i.e., $\gamma_{\mathrm{A}}^{ \pm} \neq \gamma_{\mathrm{B}}^{ \pm}$.

According to the Arrhenius law, the rate constants are written in the exponential form: a negative exponent is equal to the ratio of the energy barrier to be surmounted by the particle to the thermal energy $k_{\mathrm{B}} T$ ( $k_{\mathrm{B}}$ is the Boltzmann constant and $T$ is the absolute temperature) and a pre-exponent (defined for various motion regimes in terms of the Kramers theory ${ }^{24}$ ) determines an attempt frequency of barrier-crossing. We can thus present the multiplicative form of the rate constants concerned (see Appendix 2):

$$
\begin{aligned}
& \alpha_{\mathrm{AB}}^{ \pm}=u_{\mathrm{A}}^{ \pm} v_{\alpha}^{ \pm} \varphi_{\mathrm{A} \alpha}, \quad \alpha_{\mathrm{BA}}^{ \pm}=u_{\mathrm{B}}^{ \pm} v_{\alpha}^{ \pm} \varphi_{\mathrm{B} \alpha}, \\
& \beta_{\mathrm{AB}}^{ \pm}=u_{\mathrm{A}}^{ \pm} v_{\beta}^{ \pm} \varphi_{\mathrm{A} \beta}, \quad \beta_{\mathrm{BA}}^{ \pm}=u_{\mathrm{B}}^{ \pm} v_{\beta}^{ \pm} \varphi_{\mathrm{B} \beta}
\end{aligned}
$$

We use the shorthand symbols for combinations of model parameters, each having clear physical meaning: $v_{\alpha}^{ \pm}$and $v_{\beta}^{ \pm}$are determined solely by the maxima of the potential profile for the conformations + and - , which correspond to the barriers $\alpha$ and $\beta ; u_{\mathrm{A}}^{ \pm}$and $u_{\mathrm{B}}^{ \pm}$are specified by the minima of potential wells $\mathrm{A}$ and $\mathrm{B}$ and by some other well parameters (their curvature etc.); $\varphi$ account for the effect of external forces (a load force or a concentration gradient) on the transition rate constants in the conformations + and - (they are labeled by a pair of indices for the certain potential well and the neighboring potential barrier). As an example, the parameters $u_{\mathrm{A}}^{ \pm}$and $v_{\alpha}^{ \pm}$ designate $k_{\mathrm{A}}^{ \pm} \exp \left(U_{\mathrm{A}}^{ \pm} / k_{\mathrm{B}} T\right)$ and $\exp \left(-U_{\alpha}^{ \pm} / k_{\mathrm{B}} T\right)$, respectively, where $U_{\mathrm{A}}^{ \pm}$and $U_{\alpha}^{ \pm}$are the minimum and the maximum of the \pm potential profile for well $\mathrm{A}$ and barrier $\alpha$ [for simplicity, we assume that the pre-exponents are unchanged under conformational transformations, i.e., $k_{\mathrm{A}}^{+}=k_{\mathrm{A}}^{-}$and $k_{\mathrm{B}}^{+}=k_{\mathrm{B}}^{-}$(see the last paragraph in Appendix 2)]. As an example for the parameter $\varphi$, consider $\varphi_{\mathrm{A \alpha}}=\exp \left(-F l_{\mathrm{A \alpha}} / k_{\mathrm{B}} T\right)$, where $-F$ is the load force and $l_{\mathrm{A} \alpha}=x_{\alpha}-x_{\mathrm{A}}$, where $x_{\mathrm{A}}$ and $x_{\alpha}$ are the positions of the well A bottom and the barrier $\alpha$ top on the $x$-axis. As is seen, the parameter $\varphi_{\mathrm{A \alpha}}$ represents a reduction of the rate constants $\alpha_{\mathrm{AB}}^{ \pm}$due to load-force-induced increase in the corresponding potential barrier height. So in shorthand form, the factor $\alpha_{\mathrm{AB}}^{ \pm}$ $\beta_{\mathrm{BA}}^{ \pm}-\alpha_{\mathrm{BA}}^{ \pm} \beta_{\mathrm{AB}}^{ \pm}$, which is a direct measure of detailed balance breaking in either conformation (see eq 6), can be written as

$$
\alpha_{\mathrm{AB}}^{ \pm} \beta_{\mathrm{BA}}^{ \pm}-\alpha_{\mathrm{BA}}^{ \pm} \beta_{\mathrm{AB}}^{ \pm}=u_{\mathrm{A}}^{ \pm} u_{\mathrm{B}}^{ \pm} v_{\alpha}^{ \pm} v_{\beta}^{ \pm} \varphi_{\mathrm{B} \alpha} \varphi_{\mathrm{A} \beta}\left[\exp \left(-F L / k_{\mathrm{B}} T\right)-1\right]
$$

Here we have used the equalities $\varphi_{\alpha \mathrm{B}}=\left(\varphi_{\mathrm{B} \alpha}\right)^{-1}, \varphi_{\beta \mathrm{A}}=\left(\varphi_{\mathrm{A} \beta}\right)^{-1}$, and $\varphi_{\mathrm{A} \alpha} \varphi_{\alpha \mathrm{B}} \varphi_{\mathrm{B} \beta} \varphi_{\beta \mathrm{A}}=\exp \left(-F L / k_{\mathrm{B}} T\right)$, where $L=l_{\mathrm{A} \alpha}+l_{\alpha \mathrm{B}}+$ $l_{\mathrm{B} \beta}+l_{\beta \mathrm{A}}$ is the potential period. As follows from eq 10 , in the unloaded regime, $F=0$, there is equilibrium in either conformation (but not in the whole system) (cf. eq 6).

Consider the unloaded regime in more detail. First, note that all parameters $\varphi$ become unity and expressions 3 and 4 for the fluxes $J$ and $J_{\mathrm{r}}$ are significantly simplified on substituting the $\alpha$ - and $\beta$-channel rate constants in multiplicative form 9:

$$
\begin{aligned}
J= & \Delta^{-1}\left(v_{\alpha}^{+} v_{\beta}^{-}-v_{\alpha}^{-} v_{\beta}^{+}\right) u_{\mathrm{B}}^{+} u_{\mathrm{A}}^{-} \gamma_{\mathrm{A}}^{+} \gamma_{\mathrm{B}}^{-}\left[\exp \left(\mu_{\mathrm{S}} / k_{\mathrm{B}} T\right)-1\right] \\
J_{\mathrm{r}}= & \Delta^{-1}\left(v_{\alpha}^{+}+v_{\beta}^{+}\right)\left(v_{\alpha}^{-}+v_{\beta}^{-}\right) u_{\mathrm{B}}^{+} u_{\mathrm{A}}^{-} \gamma_{\mathrm{A}}^{+} \gamma_{\mathrm{B}}^{-}\left[\exp \left(\mu_{\mathrm{S}} / k_{\mathrm{B}} T\right)-1\right] \\
\Delta= & \sum_{\sigma^{\prime} \neq \sigma= \pm}\left\{( u _ { \mathrm { A } } ^ { \sigma ^ { \prime } } + u _ { \mathrm { B } } ^ { \sigma ^ { \prime } } ) ( v _ { \alpha } ^ { \sigma ^ { \prime } } + v _ { \beta } ^ { \sigma ^ { \prime } } ) \left[\left(v_{\alpha}^{\sigma}+v_{\beta}^{\sigma}\right) \times\right.\right. \\
& \left.\left(u_{\mathrm{B}}^{\sigma} \gamma_{\mathrm{A}}^{\sigma}+u_{\mathrm{A}}^{\sigma} \gamma_{\mathrm{B}}^{\sigma}\right)+\gamma_{\mathrm{A}}^{\sigma} \gamma_{\mathrm{B}}^{\sigma}\right]+\left[u_{\mathrm{A}}^{\sigma}\left(v_{\alpha}^{\sigma}+v_{\beta}^{\sigma}\right)+\right. \\
& \left.\left.u_{\mathrm{B}}^{\sigma^{\prime}}\left(v_{\alpha}^{\sigma^{\prime}}+v_{\beta}^{\sigma^{\prime}}\right)\right] \gamma_{\mathrm{A}}^{\sigma^{\prime}} \gamma_{\mathrm{B}}^{\sigma}\right\}
\end{aligned}
$$

Here we have introduced the quantity

$$
\mu_{\mathrm{S}}=k_{\mathrm{B}} T \ln \left(\frac{u_{\mathrm{A}}^{+} u_{\mathrm{B}}^{-} \gamma_{\mathrm{B}}^{+} \gamma_{\mathrm{A}}^{-}}{u_{\mathrm{B}}^{+} u_{\mathrm{A}}^{-} \gamma_{\mathrm{A}}^{+} \gamma_{\mathrm{B}}^{-}}\right)
$$

which, as demonstrated in Appendix 2, specifies the chemical potential of the unbalance source.

The meaning of $\mu_{\mathrm{S}}$ becomes more clear when it is written in terms of the model parameters:

$$
\mu_{\mathrm{S}}=U_{\mathrm{B}}^{-}-U_{\mathrm{B}}^{+}-U_{\mathrm{A}}^{-}+U_{\mathrm{A}}^{+}+k_{\mathrm{B}} T \ln \frac{\gamma_{\mathrm{B}}^{+} \gamma_{\mathrm{A}}^{-}}{\gamma_{\mathrm{A}}^{+} \gamma_{\mathrm{B}}^{-}}
$$

The equilibrium state implies thermal noise driven transitions only, so that the interconformational rate constant ratios are $\gamma_{\mathrm{A}(\mathrm{B})}^{+}$ $\gamma_{\mathrm{A}(\mathrm{B})}^{-}=\exp \left[\left(-U_{\mathrm{A}(\mathrm{B})}^{+}+U_{\mathrm{A}(\mathrm{B})}^{-}\right) / k_{\mathrm{B}} T\right]$ and hence at equilibrium $\mu_{\mathrm{S}}$ $=0$. Equation 11 shows that both the reciprocating and directed fluxes are proportional to $\left[\exp \left(\mu_{\mathrm{S}} / k_{\mathrm{B}} T\right)-1\right]$. So nonzero values of $\mu_{\mathrm{S}}$ destroy the system equilibrium and determine the direction and magnitude of the emerging fluxes. The sum $\left(U_{\mathrm{B}}^{-}-U_{\mathrm{B}}^{+}-\right.$ $U_{\mathrm{A}}^{-}+U_{\mathrm{A}}^{+}$) in eq 13 is the energetic contribution due to fluctuations of well minima, and the last term in this equation can be associated with the entropic contribution induced by the state dependence of the rate constants. This suggests two mechanisms by which the motion occurs: the energetic mechanism, in which the energy fed into the system in each transition from one state to the other is converted into the particle potential energy and then used to push the particle forward or back upon relaxation, and the informational mechanism, which makes nonequilibrium noise act as a source or sink of physical information about the particle position. The observation that there are two quite different mechanisms underlying the model operation is one of the main findings of this work. Noteworthy is that the concept of two mechanisms (energetic and informational) has been often used to study noise-induced transport in several contexts. ${ }^{9,13,25-28}$ Here we focus not only on directed motion but also on the reciprocating motion generation. 
In the unloaded regime, the flux ratio giving motion rectification coefficient, eq 2 , reads

$$
\kappa=\frac{v_{\alpha}^{+} v_{\beta}^{-}-v_{\alpha}^{-} v_{\beta}^{+}}{\left(v_{\alpha}^{+}+v_{\beta}^{+}\right)\left(v_{\alpha}^{-}+v_{\beta}^{-}\right)}
$$

The fact that the rectification coefficient depends solely on barrier parameters is another one of the main results of the present paper. As eq 14 indicates, (i) $|\kappa| \leq 1$, as it should be, and (ii) the motion rectification is only possible provided that barrier fluctuations are asymmetric. The rectification coefficient rises together with fluctuation asymmetry. For the $\kappa$ value to reach its maximum (unity), one of the barriers should be infinitely high and its position should fluctuate by half a period of the potential profile $\left(v_{\beta}^{+}=0, v_{\alpha}^{-}=0\right)$. Then the reverse particle flux is blocked in either conformation.

The important role of externally induced barrier fluctuations was first recognized in studies of enzymatic catalysis ${ }^{14}$ and the active transport of ions through cell membranes. ${ }^{29}$ The idea that directed motion can be provided by appropriately raising and lowering potential barriers and wells, with fluctuating barriers acting as a series of gates, was later discussed in various contexts. ${ }^{3,26}$ A different approach to exploit asynchronously oscillating barriers for directed transport has been recently suggested. ${ }^{30}$ The present work focuses on the rectifying effect of asymmetric fluctuations of potential barriers. Importantly, eq 14 holds true not only for the energy-driven motion mechanism induced by potential well fluctuations but also in the case similar to "chemical Maxwell demon" ${ }^{31}$ when the motion is caused by the informational mechanism.

Now let us return to the general case, with nonzero value of the load force, and discuss efficiency of energy conversion. Alongside with the widely accepted efficiency definition based on the thermodynamic consideration, ${ }^{31,32}$ various alternative efficiencies (Stokes efficiency, ${ }^{33}$ efficiency at maximum power output, ${ }^{34}$ Peclet number, ${ }^{35}$ etc.) and related quantities (like entropy production) have been introduced and discussed in the literature. ${ }^{36} \mathrm{We}$ follow the thermodynamic definition: the efficiency is given by the ratio of the mechanical work per unit time done by the motor against the external force (power output) to the free energy supplied per time unit by the source of nonequilibrium (power input). In our model, the power output is $P_{\text {out }}=F L J$ and the power input is $P_{\text {in }}=\mu_{\mathrm{S}} J_{\mathrm{r}}$ (see eq A2.7 in Appendix 2), so that the efficiency reads

$$
\eta=\frac{F L J}{\mu_{\mathrm{S}} J_{\mathrm{r}}}=\frac{F L}{\mu_{\mathrm{S}}} \kappa
$$

Note that $\eta$ is proportional to the rectification coefficient, as one would expect. Generally, the expression for the efficiency, eq 15 , is a rather complicated function of the model parameters. A particular simple case, which can be treated easily, is that where one of the barriers is infinitely high, so that $v_{\beta}^{+}=v_{\alpha}^{-}=$ 0 , the fluxes $J$ and $J_{\mathrm{r}}$ are equal and proportional to $\exp \left[\left(\mu_{\mathrm{S}}-\right.\right.$ $\left.F L) / k_{\mathrm{B}} T\right]-1$, and the rectification coefficient becomes unity. As the load force rises from zero to $F_{\mathrm{s}}$, where $F_{\mathrm{s}}=\mu_{\mathrm{S}} / L$ is the stopping force in this case, the energy conversion efficiency linearly increases and approaches unity in the vicinity of $F_{\mathrm{s}}$. A detailed analysis of the efficiency near the stopping point can be performed in the limit of high (but not infinitely high) barrier in a way similar to that proposed for the case with stateindependent rate constants. ${ }^{21}$

\section{The Antisymmetric Case}

In order to obtain explicit expressions for the quantities of interest, we reduce a number of independent parameters by taking advantage of the antisymmetric version of the model. Note that the antisymmetric model is characterized by the highest energy conversion efficiency. ${ }^{21,23}$ Within the antisymmetric model, wells A and B have the same curvatures in the vicinity of their minima which are equidistant by $L / 4$ from neighboring maxima; the potential profiles of the + and conformations are mutually shifted by half a period $L$ (see Figure 2). The above-introduced parameters are concretized for the antisymmetric case as follows:

$$
\begin{gathered}
u_{\mathrm{A}}^{+}=u_{\mathrm{B}}^{-}=k_{0} e^{u}, \quad u_{\mathrm{B}}^{+}=u_{\mathrm{A}}^{-}=k_{0} e^{-u}, \\
v_{\alpha}^{+}=v_{\beta}^{-}=e^{-v}, v_{\beta}^{+}=v_{\alpha}^{-}=e^{-V}, \\
\varphi_{\mathrm{A} \alpha}=\varphi_{\alpha \mathrm{B}}=\varphi_{\mathrm{B} \beta}=\varphi_{\beta \mathrm{A}}=e^{-f}, \\
f=F L / 4 k_{\mathrm{B}} T, u+f<v<V, \\
\gamma_{\mathrm{A}}^{-}=\gamma_{\mathrm{B}}^{+}, \gamma_{\mathrm{B}}^{-}=\gamma_{\mathrm{A}}^{+}, \zeta=k_{0} e^{-v} / \sqrt{\gamma_{\mathrm{B}}^{+} \gamma_{\mathrm{A}}^{+}}
\end{gathered}
$$

The parameter $k_{0}$ characterizes the oscillation frequency of a particle in the potential well; $u, v$, and $V$ are the energies of potential minima and maxima in units of $k_{\mathrm{B}} T ; f$ is a change in potential barrier heights caused by a load force; the parameter $\zeta$ determines the average number of low-barrier crossings during the average lifetime of the + and - conformations. The inequality $f<v-u$ restricts the values of the force applied such that the positions of potential extrema remain unchanged. The state-dependent rate constants $\gamma_{\mathrm{A}}^{ \pm}$and $\gamma_{\mathrm{B}}^{ \pm}$are assumed to be antisymmetrized as well. Substituting eq 16 into eqs 3, 4, and 15 , we get

$$
\begin{aligned}
& \sinh (w-f)-e^{-V+v}[\sinh (w+f)+ \\
& J=k_{0} e^{-v} \frac{2 \xi \sinh (2 f)]}{\left(\gamma_{\mathrm{A}}^{+}+\gamma_{\mathrm{B}}^{+}\right) / \sqrt{\gamma_{\mathrm{A}}^{+} \gamma_{\mathrm{B}}^{+}}+2 \xi[\cosh (u-f)+} \\
& \left.e^{-V+v} \cosh (u+f)\right] \\
& \sinh (w-f)-e^{-V+v}[\sinh (w+f)+ \\
& \kappa=\frac{2 \zeta \sinh (2 f)]}{\sinh (w-f)+e^{-V+v} \sinh (w+f)}, \quad \eta=\frac{f}{w} \kappa
\end{aligned}
$$

where

$$
w=\frac{\mu_{\mathrm{S}}}{4 k_{\mathrm{B}} T}=u+\frac{1}{2} \ln \left(\frac{\gamma_{\mathrm{B}}^{+}}{\gamma_{\mathrm{A}}^{+}}\right)
$$

As eq 17 indicates, $\kappa \leq 1$, in accordance with its physical meaning.

These expressions generalize eqs 12 and 21 of our previous work ${ }^{23}$ to the case of position-dependent rate constants for transitions between the + and - conformations. The principal result of such generalization is an additional mechanism of noise-induced motion. Indeed, if the conformational transitions rate constants are state-independent, $\gamma_{\mathrm{A}}^{+}=\gamma_{\mathrm{B}}^{+}=\gamma$, there is the only contribution $u=u_{0}$ in $w$, eq 18 , due to fluctuations of potential well minima. In this particular case the motor operates via the energetic mechanism and eqs 17 and 18 reproduce the results obtained for the simplified model. ${ }^{23}$ However, the model under consideration generates directed motion even without the energy fluctuations, $u=0$, in contrast to the simplified model. The rectification coefficient and the efficiency (but not the fluxes) are the same, as in the previous case, provided $\gamma_{\mathrm{A}}^{+}=\gamma$ $\exp \left(-u_{0}\right)$ and $\gamma_{\mathrm{B}}^{+}=\gamma \exp \left(u_{0}\right)$. This occurs due to fluctuations of the state-dependent rate constants responsible for interconformation transitions (informational mechanism). Generally, 


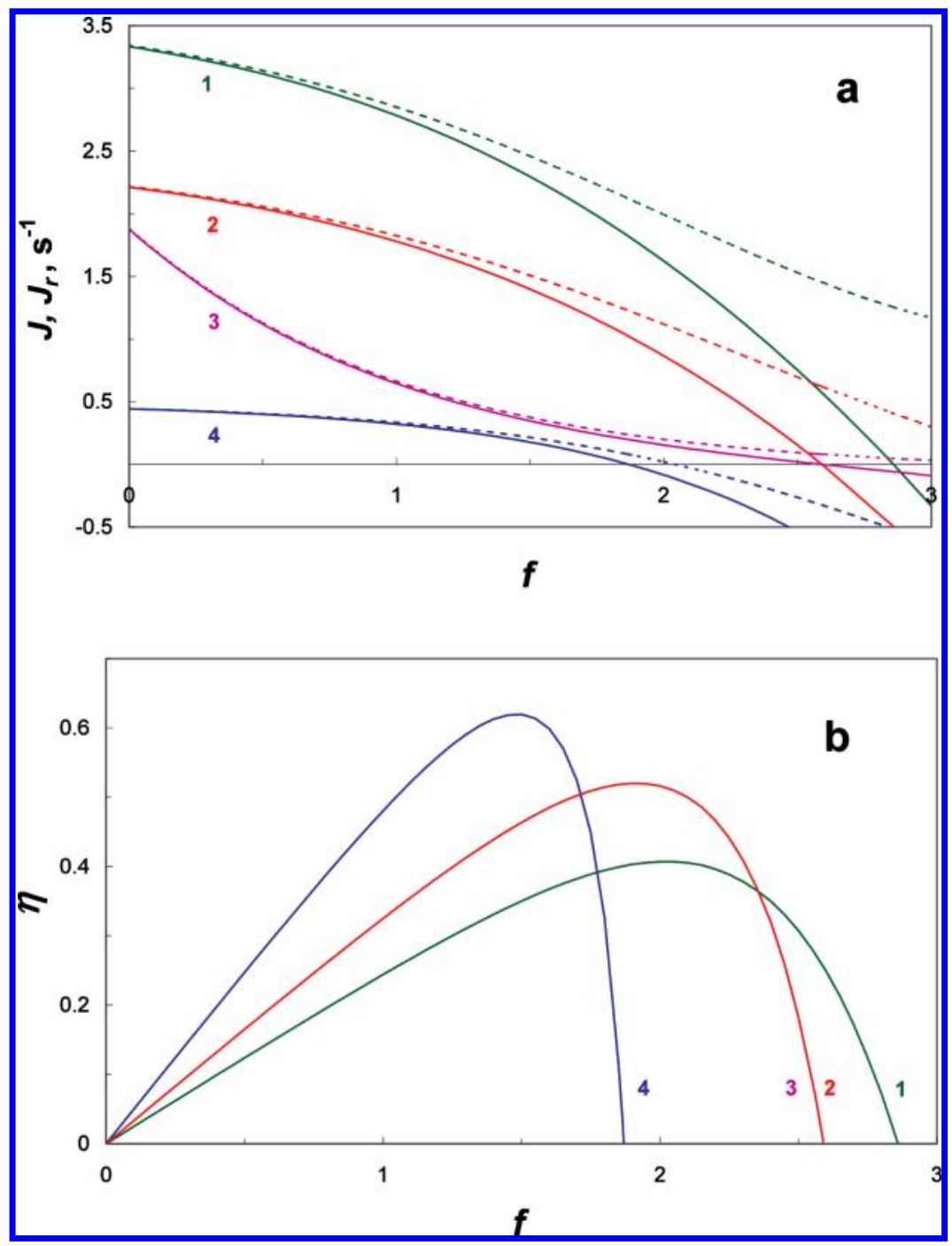

Figure 3. Net circulation (solid lines) and reciprocating (dashed lines) fluxes (panel a) as well as efficiency (panel b) versus the load force (see eq 17). For curves 1,2, and 4: $u=3$ and $\gamma_{\mathrm{A}}^{+}=1 \mathrm{~s}^{-1}, \gamma_{\mathrm{B}}^{+}=e^{2} \approx 7.39 \mathrm{~s}^{-1}$ (curve 1); $\gamma_{\mathrm{A}}^{+}=\gamma_{\mathrm{B}}^{+}=5 \mathrm{~s}^{-1}$ (curve 2), $\gamma_{\mathrm{A}}^{+} \approx 7.39 \mathrm{~s}^{-1}, \gamma_{\mathrm{B}}^{+}=1 \mathrm{~s}^{-1}(\operatorname{curve}$ 4). For curve $3, u=0$ and $\gamma_{\mathrm{A}}^{+}=5 e^{-3} \approx 0.25 \mathrm{~s}^{-1}, \gamma_{\mathrm{B}}^{+}=5 e^{3} \approx 100 \mathrm{~s}^{-1}$. Curves 2 and 3 , with the same values of $w=3$ and $\sqrt{ }\left[\gamma_{\mathrm{A}}^{+} \gamma_{\mathrm{B}}^{+}\right]=5$, represent the energetic $\left(\gamma_{\mathrm{A}}^{+}=\gamma_{\mathrm{B}}^{+}\right)$and the informational $(u=0)$ mechanisms. For all curves $V=12, v=5.5, k_{0}=1000 \mathrm{~s}^{-1}$.

when $u \neq 0$ and $\gamma_{\mathrm{A}}^{+} \neq \gamma_{\mathrm{B}}^{+}$, there are two contributions in the driving force (see the previous section), and both mechanisms, energetic and informational, are operative.

Figure 3a demonstrates the characteristic dependences of both fluxes on the load force at various values of model parameters. One can see that the directed flux is always less than the reciprocating one and hence its positivity region lies entirely within that of the latter. As the entropic contribution increases and the system moves further away from equilibrium (i.e., as the ratio $\gamma_{\mathrm{B}}^{+} / \gamma_{\mathrm{A}}^{+}$rises together with the parameter $w$ varying from 2 to 4 for curves $4,2,1$ ), the fluxes are enhanced whereas the efficiency is reduced (Figure 3, a and b). It is interesting to compare curves 2 and 3 which represent the physically important limiting cases, where only one from the motion-inducing mechanisms is operative. Both of them are calculated for the same values of $w$ and $\sqrt{ }\left[\gamma_{\mathrm{A}}^{+} \gamma_{\mathrm{B}}^{+}\right]$, but in the former case we have taken $u=3$ and identical rate constants $\gamma_{\mathrm{A}}^{+}$and $\gamma_{\mathrm{B}}^{+}$, while in the latter these parameters are $u=0$ and $\gamma_{\mathrm{A}}^{+} \neq \gamma_{\mathrm{B}}^{+}$. So curves 2 and 3 can be associated with the energetic and information mechanism, respectively. These curves coincide in Figure 3b, but they are different from each other in Figure 3a. In the case of the energetic mechanism, the plots of $J(f)$ and $J_{\mathrm{r}}(f)$ exhibit a negative curvature. In contrast, the corresponding curves for the informational mechanism are concave functions of $f$. For the parameter values chosen, curve 2 in Figure 3 a lies higher than curve 3 at all $f$; however, generally, the answer on the question which mechanism is preferable for the current (at given value of the efficiency) depends on particular values of the model parameters. For example, at $f=0$ and $\zeta>[1+\exp (-V$ $+v)$ ] the informational mechanism provides higher velocity of motion than energetic one.

In the unloaded regime $(f=0)$, the rectification coefficient takes a particularly simple form: $\kappa=\tanh [(V-v) / 2]$. It decreases monotonically with the rising load force and becomes zero at $f=f_{\mathrm{s}}$, where $f_{\mathrm{s}}$ is the stopping force defined by a root of the numerator of $J$ (or $\kappa)$ in eq 17. At $\zeta \exp (-V+v) \gg 1$ (i.e., when the values of $\gamma_{\mathrm{A}}^{+} \gamma_{\mathrm{B}}^{+}$are small enough), we obtain $f_{\mathrm{s}}$ $\approx(4 \zeta)^{-1}[\exp (V-v)-1] \sinh w$. If the inverse inequality holds together with the condition $\varepsilon \equiv 2 \mathrm{e}^{-V+v} \sinh (2 w) / w \ll 1$, then the stopping point of the motor is determined by the approximate relation $f_{\mathrm{s}} \approx[1-(1 / 2+\xi) \varepsilon] w$. As far as the kinetic approach is concerned, the interconformation transitions should be slow enough, which implies that $\sqrt{ }\left[\gamma_{\mathrm{A}}^{+} \gamma_{\mathrm{B}}^{+}\right]$is lesser than the inverse time $D / L^{2}$ of the particle diffusion over distances of the order $L$ (where $D$ is the diffusion coefficient). Nevertheless, this restriction allows for values $\xi \ll 1$ if $k_{0} e^{-v} \ll \sqrt{ }\left[\gamma_{\mathrm{A}}^{+} \gamma_{\mathrm{B}}^{+}\right] \ll D / L^{2}$. 
On the condition $V>v+2 w$, the reciprocating flux $J_{\mathrm{r}}=J / \kappa$ vanishes at the point $f_{0}$ specified by the equation $\tanh f_{0}=\tanh$ $w / \tanh [(V-v) / 2]$. Since it is always so that $f_{\mathrm{s}}<w<f_{0}$, both fluxes and the efficiency are positive in the operational region of the motor $\left(0 \leq f<f_{\mathrm{s}}\right)$. As already mentioned, the maximum efficiency is reached near the stopping force $f_{\mathrm{s}}$. In this region, the efficiency can be estimated at $\varepsilon \ll 1$ and $\varepsilon \zeta \ll 1$ using the approximate expression

$$
\eta \approx \frac{f}{w} \frac{f_{\mathrm{s}}-f}{f_{0}-f}
$$

which suggests that the maximum of the efficiency is given by

$$
\eta_{\mathrm{m}} \approx 1-2 \sqrt{(1+\zeta)} \varepsilon, \quad f_{\mathrm{m}} \approx[1-\sqrt{(1+\zeta)} \varepsilon] w<f_{\mathrm{s}}
$$

These asymptotic results confirm the above-stated regularity: high efficiency is implemented provided the barrier height $V$ is sufficient to block the reverse flux. Efficiency is the higher, the smaller the parameter $w$ and the closer the system to the equilibrium state. We emphasize again that such effect results from the specificity of the two-well potential profile fluctuating by half a period and containing a sufficiently high barrier to rule out the reverse motion. An alternative high-efficiency mechanism acting far from equilibrium is typical of strongly asymmetric potentials shaped identically on both half-periods which are largely shifted in energy relative to each other. ${ }^{37}$ In terms of the kinetic approach, this mechanism is probable at large values of parameters $V, v$, and $w$, mutually related as $V=$ $v+2 w$.

\section{Conclusions}

This paper is devoted to the development of the theory of two-step energy conversion into directed mechanical motion on the nanoscale: first, a part of the free energy coming from a source of nonequilibrium is converted to induced reciprocating motion, which is then rectified by a symmetry-breaking mechanism. To illustrate this approach, we have considered a simple kinetic model, in which the system fluctuates between two states through two reaction channels with fluctuating transition rates. The distinctive feature of the model is that not only the intraconformational, but also the interconformational rate constants are position dependent. A notable observation is that the generalized driving force of the reciprocating motion is caused by two sources: the energy contribution due to energy fluctuations between states (the energetic mechanism) and the entropic contribution due to fluctuations of state-dependent transition rate constants (the informational mechanism). The induced reciprocating motion is rectified by the asymmetric fluctuations of reaction-channel rate constants or, in terms of the flashing potential model, of potential barriers. For the antisymmetric version of the model, simple explicit expressions have been derived for reciprocating and net fluxes as well as for the efficiency with which the energy of nonequilibrium fluctuations is converted to useful work. As the system moves further away from equilibrium, both reciprocating and net circulation fluxes are enhanced whereas the efficiency is reduced. The highest efficiency values are reached in the vicinity of the stopping point when one of the fluctuating barriers is much higher than the other and blocks the reverse flux. The present approach offers more generality than those employed before, as it extends all the relationships relevant to directed motion emergence (including the conditions for high-efficiency motor operation) to the informational mechanism as well.
Acknowledgment. The authors thank M. L. Dekhtyar for helpful comments on the manuscript. This work was supported by Academia Sinica. Yu.A.M. thanks the Russian Foundation for Basic Research for partial support (grant no. 10-03-00393). Yu.A.M. and V.M.R. gratefully acknowledge the kind hospitality received from the Institute of Atomic and Molecular Sciences.

\section{Appendix 1}

Within the kinetic approach, a master equation for the probabilities $\rho_{\mathrm{A}(\mathrm{B})}^{ \pm}$to find the system in states $\mathrm{A}^{ \pm}\left(\mathrm{B}^{ \pm}\right)$can be written in the form

$$
\frac{\mathrm{d}}{\mathrm{d} t}\left(\begin{array}{c}
\rho_{\mathrm{A}}^{+} \\
\rho_{\mathrm{B}}^{+} \\
\rho_{\mathrm{A}}^{-} \\
\rho_{\mathrm{B}}^{-}
\end{array}\right)=\left(\begin{array}{c}
J_{\mathrm{A}}+J_{\beta}^{+}-J_{\alpha}^{+} \\
J_{\alpha}^{+}-J_{\beta}^{+}-J_{\mathrm{B}} \\
J_{\beta}^{-}-J_{\alpha}^{-}-J_{\mathrm{A}} \\
J_{\mathrm{B}}+J_{\alpha}^{-}-J_{\beta}^{-}
\end{array}\right)
$$

where the fluxes entering into the right-hand side of the matrix equation are shown in Figure $1 \mathrm{~b}$ and defined by eq 1 . With these definitions and notation 5, we obtain the system of equations for the steady-state regime $\left(\mathrm{d} \rho_{\mathrm{A}(\mathrm{B})}^{ \pm} / \mathrm{d} t=0\right)$ :

$$
\left(\begin{array}{cccc}
-\xi_{\mathrm{A}}^{+}-\gamma_{\mathrm{A}}^{+} & -\xi_{\mathrm{B}}^{+} & \gamma_{\mathrm{A}}^{-} & 0 \\
\xi_{\mathrm{A}}^{+} & -\xi_{\mathrm{B}}^{+}-\gamma_{\mathrm{B}}^{+} & 0 & \gamma_{\mathrm{B}}^{-} \\
\gamma_{\mathrm{A}}^{+} & 0 & -\xi_{\mathrm{A}}^{-}-\gamma_{\mathrm{A}}^{-} & \xi_{\mathrm{B}}^{-} \\
0 & \gamma_{\mathrm{B}}^{+} & \xi_{\mathrm{A}}^{-} & -\xi_{\mathrm{B}}^{-}-\gamma_{\mathrm{B}}^{-}
\end{array}\right)\left(\begin{array}{c}
\rho_{\mathrm{A}}^{+} \\
\rho_{\mathrm{B}}^{+} \\
\rho_{\mathrm{A}}^{-} \\
\rho_{\mathrm{B}}^{-}
\end{array}\right)=0
$$

Taking into account the normalization condition

$$
\rho_{\mathrm{A}}^{+}+\rho_{\mathrm{B}}^{+}+\rho_{\mathrm{A}}^{-}+\rho_{\mathrm{B}}^{-}=1
$$

the solution of eq A1.2 appears as

$$
\left(\begin{array}{c}
\rho_{\mathrm{A}}^{+} \\
\rho_{\mathrm{B}}^{+} \\
\rho_{\mathrm{A}}^{-} \\
\rho_{\mathrm{B}}^{-}
\end{array}\right)=\Delta^{-1}\left(\begin{array}{l}
\xi_{\mathrm{B}}^{+} \xi_{\mathrm{B}}^{-} \gamma_{\mathrm{A}}^{-}+\xi_{\mathrm{B}}^{+} \xi_{\mathrm{A}}^{-} \gamma_{\mathrm{B}}^{-}+\xi_{\mathrm{B}}^{-} \gamma_{\mathrm{B}}^{+} \gamma_{\mathrm{A}}^{-}+\xi_{\mathrm{B}}^{+} \gamma_{\mathrm{A}}^{-} \gamma_{\mathrm{B}}^{-} \\
\xi_{\mathrm{A}}^{+} \xi_{\mathrm{B}}^{-} \gamma_{\mathrm{A}}^{-}+\xi_{\mathrm{A}}^{+} \xi_{\mathrm{A}}^{-} \gamma_{\mathrm{B}}^{-}+\xi_{\mathrm{A}}^{-} \gamma_{\mathrm{A}}^{+} \gamma_{\mathrm{B}}^{-}+\xi_{\mathrm{A}}^{+} \gamma_{\mathrm{A}}^{-} \gamma_{\mathrm{B}}^{-} \\
\xi_{\mathrm{B}}^{+} \xi_{\mathrm{B}}^{-} \gamma_{\mathrm{A}}^{+}+\xi_{\mathrm{A}}^{+} \xi_{\mathrm{B}}^{-} \gamma_{\mathrm{B}}^{+}+\xi_{\mathrm{B}}^{+} \gamma_{\mathrm{A}}^{+} \gamma_{\mathrm{B}}^{-}+\xi_{\mathrm{B}}^{-} \gamma_{\mathrm{A}}^{+} \gamma_{\mathrm{B}}^{+} \\
\xi_{\mathrm{B}}^{+} \xi_{\mathrm{A}}^{-} \gamma_{\mathrm{A}}^{+}+\xi_{\mathrm{A}}^{+} \xi_{\mathrm{A}}^{-} \gamma_{\mathrm{B}}^{+}+\xi_{\mathrm{A}}^{-} \gamma_{\mathrm{A}}^{+} \gamma_{\mathrm{B}}^{+}+\xi_{\mathrm{A}}^{+} \gamma_{\mathrm{B}}^{+} \gamma_{\mathrm{A}}^{-}
\end{array}\right)
$$

where

$$
\begin{array}{r}
\Delta=\sum_{\sigma^{\prime} \neq \sigma= \pm}\left[\left(\xi_{\mathrm{A}}^{\sigma^{\prime}}+\xi_{\mathrm{B}}^{\sigma^{\prime}}\right)\left(\xi_{\mathrm{B}}^{\sigma} \gamma_{\mathrm{A}}^{\sigma}+\xi_{\mathrm{A}}^{\sigma} \gamma_{\mathrm{B}}^{\sigma}+\gamma_{\mathrm{A}}^{\sigma} \gamma_{\mathrm{B}}^{\sigma}\right)+\left(\xi_{\mathrm{A}}^{\sigma}+\right.\right. \\
\left.\left.\xi_{\mathrm{B}}^{\sigma^{\prime}}\right) \gamma_{\mathrm{A}}^{\sigma^{\prime}} \gamma_{\mathrm{B}}^{\sigma}\right]
\end{array}
$$

Substituting solution A1.4 into the definitions of the fluxes desired we arrive at eqs 3 and 4 .

\section{Appendix 2}

The reduction of the continuous description to a kinetic one implies that the particle motion dynamics can be subdivided into a fast and a slow component. ${ }^{38}$ The separation of the two time scales is possible if a particle moves in the potential with interwell barrier heights exceeding the particle thermal energy $k_{\mathrm{B}} T$ and if potential fluctuation frequencies are much less than the inverse time $D / L^{2}$ of the particle diffusion over the potential period $L .{ }^{39}$ Here we provide a basis for the calculation of the motor efficiency in terms of the kinetic approach and multiplicative representation of rate constants (9). To this end, we use the method previously developed to analyze energetics of reciprocating motion on the nanoscale. ${ }^{13}$ For periodic potential 
profiles $U_{ \pm}(x)$ and position-dependent rate constants $\gamma_{ \pm}(x)$, the steady-state free energy supplied to the system per unit time by a nonequilibrium source reads

$$
P_{\text {in }}=\int_{0}^{L} \mathrm{~d} x \mu_{S}(x)\left[\gamma_{+}(x) \rho_{+}(x)-\gamma_{-}(x) \rho_{-}(x)\right]
$$

Here the chemical potential of the "effective fuel" is ${ }^{13}$

$$
\mu_{\mathrm{S}}(x)=\mu_{-}^{0}-\mu_{+}^{0}+U_{-}(x)-U_{+}(x)+k_{\mathrm{B}} T \ln \frac{\gamma_{+}(x)}{\gamma_{-}(x)}
$$

with $\mu_{ \pm}^{0}=\mu_{ \pm}^{0}(T)$ denoting position-independent standard chemical potentials, and the following conditions are adopted for normalization and integral balance:

$$
\begin{array}{r}
\int_{0}^{L} \mathrm{~d} x\left[\rho_{+}(x)+\rho_{-}(x)\right]=1, \quad \int_{0}^{L} \mathrm{~d} x\left[\gamma_{+}(x) \rho_{+}(x)-\right. \\
\left.\gamma_{-}(x) \rho_{-}(x)\right]=0
\end{array}
$$

Assume that potentials $U_{ \pm}(x)$ contain two wells per the period and interwell barriers are high relative to the thermal energy $k_{\mathrm{B}} T$. For definiteness, let the barriers be positioned at the points $x=0, L / 2, L$ and the wells on the intervals $(0, L / 2)$ and $(L / 2, L)$. We are concerned with the probabilities that the particle is in potential well A or B

$$
\rho_{\mathrm{A}}^{ \pm}=\int_{0}^{L / 2} \rho^{ \pm}(x) \mathrm{d} x, \quad \rho_{\mathrm{B}}^{ \pm}=\int_{L / 2}^{L} \rho^{ \pm}(x) \mathrm{d} x, \quad \sum_{\sigma= \pm} \sum_{i=\mathrm{A}, \mathrm{B}} \rho_{i}^{\sigma}=1
$$

If rate constants $\gamma_{ \pm}(x)$ do not change within potential wells (i.e., $\gamma_{ \pm}(x)=\gamma_{\mathrm{A}}^{ \pm}$at $0<x<L / 2$ and $\gamma_{ \pm}(x)=\gamma_{\mathrm{B}}^{ \pm}$at $\left.L / 2<x<L\right)$ and conformation lifetimes $\left[\gamma_{ \pm}(x)\right]^{-1}$ are much longer than the particle diffusion time through the potential period $L^{2} / D$, then local equilibrium can be established in either well and the distribution function is expressible in the form: ${ }^{39}$

$$
\begin{aligned}
& \rho_{ \pm}(x) \approx \\
& \begin{cases}\rho_{\mathrm{A}}^{ \pm} \exp \left(-\tilde{U}_{ \pm}(x) / k_{\mathrm{B}} T\right) / \int_{0}^{L / 2} \mathrm{~d} x^{\prime} \exp \left(-\tilde{U}_{ \pm}\left(x^{\prime}\right) / k_{\mathrm{B}} T\right), & 0<x<L / 2 \\
\rho_{\mathrm{B}}^{ \pm} \exp \left(-\tilde{U}_{ \pm}(x) / k_{\mathrm{B}} T\right) / \int_{L / 2}^{L} \mathrm{~d} x^{\prime} \exp \left(-\tilde{U}_{ \pm}\left(x^{\prime}\right) / k_{\mathrm{B}} T\right), & L / 2<x<L\end{cases}
\end{aligned}
$$

where $\tilde{U}^{ \pm}(x)=U^{ \pm}(x)+F x$ is the full potential energy of the system including the contribution from the load force $-F$. Nonequilibrium probabilities A2.4 satisfy eq A1.2 containing rate constants of the $\alpha$ and $\beta$ channels which appear as follows:

$$
\begin{gathered}
\alpha_{\mathrm{AB}}^{ \pm}=\frac{D}{\int_{L / 4}^{3 L / 4} \mathrm{e}^{\tilde{U}_{ \pm}(x) / k_{\mathrm{B}} T} \mathrm{~d} x \int_{0}^{L / 2} \mathrm{e}^{-\tilde{U}_{ \pm}(x) / k_{\mathrm{B}} T} \mathrm{~d} x}, \\
\alpha_{\mathrm{BA}}^{ \pm}=\frac{D}{\int_{\mathrm{AB}}^{3 L / 2} \mathrm{e}^{ \pm \tilde{U}_{ \pm}(x) / k_{\mathrm{B}} T} \mathrm{~d} x \int_{L / 2}^{L} \mathrm{e}^{-\tilde{U}_{ \pm}(x) / k_{\mathrm{B}} T} \mathrm{~d} x}, \\
\beta_{\mathrm{BA}}^{ \pm}=\frac{D}{\int_{-L / 4}^{L / 4} \mathrm{e}^{\tilde{U}_{ \pm}(x) / k_{\mathrm{B}} T} \mathrm{~d} x \int_{0}^{L / 2} \mathrm{e}^{-\tilde{U}_{ \pm}(x) / k_{\mathrm{B}} T} \mathrm{~d} x}, \\
\int_{-L / 4}^{L / \tilde{U}_{ \pm}(x) / k_{\mathrm{B}} T} \mathrm{~d} x \int_{-L}^{0} \mathrm{e}^{-\tilde{U}_{ \pm}(x) / k_{\mathrm{B}} T} \mathrm{~d} x
\end{gathered}
$$

and can be represented in multiplicative form 9. Thus, sufficiently slow switching and sufficiently high (compared to $k_{\mathrm{B}} T$ ) barriers provide the prerequisites for a convenient description of the model dynamics in terms of the kinetic approach (see solutions for the fluxes given by eqs 3 and 4 or 11 and 12).

To apply the kinetic method to the analysis of the energetics of the model, an additional condition has to be introduced. When the particle jumps from any potential well of one conformation to the corresponding well of the other conformation, certain energy losses occur in the relaxation of the distribution function from the initial to the final well shape. Evidently, relaxation processes can hardly be taken into account by the coarse-grained kinetic method. To eliminate the relaxation-induced energy losses, a restriction is imposed on potential shape fluctuations such that only relative shifts of the potential are allowed on switching between the + and - conformations, with the near-bottom well shapes retained. Then eqs A2.1 and A2.2 become

$$
P_{\text {in }}=\mu_{\mathrm{S}} J_{\mathrm{r}}
$$

where

$$
\begin{array}{r}
\mu_{\mathrm{S}}=\mu_{\mathrm{S}}\left(x_{\mathrm{B}}\right)-\mu_{\mathrm{S}}\left(x_{\mathrm{A}}\right)=U_{\mathrm{B}}^{-}-U_{\mathrm{B}}^{+}-U_{\mathrm{A}}^{-}+U_{\mathrm{A}}^{+}+ \\
k_{\mathrm{B}} T \ln \frac{\gamma_{\mathrm{B}}^{+} \gamma_{\mathrm{A}}^{-}}{\gamma_{\mathrm{A}}^{+} \gamma_{\mathrm{B}}^{-}}
\end{array}
$$

Since $u_{\mathrm{A}}^{ \pm}=k_{\mathrm{A}} \exp \left(U_{\mathrm{A}}^{ \pm} / k_{\mathrm{B}} T\right)$ and $u_{\mathrm{A}}^{ \pm}=k_{\mathrm{B}} \exp \left(U_{\mathrm{B}}^{ \pm} / k_{\mathrm{B}} T\right)$, eq 13 is obtained.

\section{References and Notes}

(1) Jülicher, F.; Ajdari, A.; Prost, J. Rev. Mod. Phvs. 1997, 69, 1269. 412 .

(2) Bustamante, C.; Keller, D.; Oster, G. Acc. Chem. Res. 2001, 34,

(3) Astumian, R. D.; Hänggi, P. Phvs. Todav 2002, 55, 33.

(4) Reimann, P. Phys. Rep. 2002, 361, 57.

(5) Kolomeisky, A. B.; Fisher, M. E. Annu. Rev. Phvs. Chem. 2007, 58,675 .

(6) Hänggi, P.; Marchesoni, F. Rev. Mod. Phvs. 2009, 81, 387.

(7) Howard, J. Mechanics of Motor Proteins and the Cytoskeleton; Sinauer Associates: Sunderland, MA, 2001.

(8) (a) Hille, B. Ionic Channels of Excitable Membranes; Sinauer Associates: Sunderland, MA, 1992. (b) Tsong, T. Y.; Xie, T. D. Appl. Phvs. A: Mater. Sci. Process. 2002, 75, 345.

(9) Kay, E. R.; Leigh, D. A.; Zerbetto, F. Angew. Chem. Int. Ed. 2006, 46,72 .

(10) Drexler, K. E. Nanosystems: Molecular Machinery, Manufacturing and Computation; Wiley: New York, 1992.

(11) (a) Voet, D.; Voet, J. G.; Pratt, C. W. Fundamentals of Biochemistry; Wiley: New York, 2001. (b) Qian, H. J. Math. Chem. 2000, 27, 219.

(12) Makhnovskii, Yu. A.; Rozenbaum, V. M.; Yang, D.-Y.; Lin, S. H.; Tsong, T. Y. Eur. Phvs. J. B 2006, 52, 501.

(13) (a) Makhnovskii, Yu. A.; Rozenbaum, V. M.; Yang, D.-Y.; Lin, S. H. J. Phvs. Chem. A 2007, 111, 9486. (b) Makhnovskii, Yu. A.; Rozenbaum, V. M.; Yang, D.-Y.; Lin, S. H. J. Chem. Phvs. 2009, 130, 164101.

(14) (a) Westerhoff, H. V.; Tsong, T. Y.; Chock, P. B.; Chen, Y.; Astumian, R. D. Proc. Natl. Acad. Sci. U.S.A. 1986, 83, 4734. (b) Astumian, R. D.; Chock, P. B.; Tsong, T. Y.; Westerhoff, H. V. Phvs. Rev. A 1989, 39, 6416. (c) Astumian, R. D.; Robertson, B. J. Chem. Phvs. 1989, 91, 4891.

(15) Astumian, R. D. J. Phvs. Chem. 1996, 100, 19075.

(16) Astumian, R. D. Proc. Natl. Acad. Sci. U.S.A. 2005, 102, 1843.

(17) (a) Fisher, M. E.; Kolomeisky, A. B. Proc. Natl. Acad. Sci. U.S.A. 2001, 98, 7748. (b) Das, R. K.; Kolomeisky, A. B. Phvs. Chem. Chem. Phys. 2009, 11, 4815.

(18) Stukalin, E. B.; Kolomeisky, A. B. J. Chem. Phvs. 2006, 124, 204901.

(19) (a) Qian, H.; Qian, M. Phvs. Rev. Lett. 2000, 84, 2271. (b) Qian, H. Biophys. Chem. 2008, 94, 263.

(20) Chemla, Y. R.; Moffitt, J. R.; Bustamante, C. J. Phvs. Chem. B 2008, 112, 6025 .

(21) Rozenbaum, V. M.; Yang, D.-Y.; Lin, S. H.; Tsong, T. Y. J.Phvs. Chem. B 2004, 108, 15880.

(22) Note that the kinetic model considered here has been analyzed before 18 in different but related context (for more detail explanation see Section II). 
(23) Makhnovskii, Yu. A.; Rozenbaum, V. M.; Yang, D.-Y.; Lin, S. H.; Tsong, T. Y. Phvs. Rev. E 2004, 69, 021102. 251.

(24) Hänggi, P.; Talkner, P.; Borkovec, M. Rev. Mod. Phys. 1990, 62,

(25) (a) Büttiker, M. Z. Phys. B 1987, 68, 161. (b) van Kampen, N. G. IBM J. Res. Dev. 1988, 32, 107. (c) Luchsinger, R. H. Phvs. Rev. E 2000, 62, 272. (d) Jayannavar, A. M. e-print cond-mat/0107079. (e) Millonas, M. M. Phvs. Rev. Lett. 1995, 74, 10.

(26) (a) Astumian, R. D.; Derényi, I. Eur. Biophys. J. 1998, 27, 474.

(b) Astumian, R. D. Phvs. Chem. Chem. Phvs. 2007, 9, 5067.

(27) (a) Qian, H. Phys. Rev. Lett. 1998, 81, 3063. (b) Wang, H. IEE Proc. Nanobiotechnol. 2003, 150, 127.

(28) (a) Sauvage, J.-P. Science 2001, 291, 2105. (b) Serreli, V.; Lee,

C.-F.; Kay, E. R.; Leigh, D. A. Nature 2007, 445, 523.

(29) Fuliński, A. Chaos 1998, 8, 549.

(30) Bleil, S.; Reimann, P.; Bechinger, C. Phvs. Rev. E 2007, 75, 031117.

(31) Parrondo, J. M. R.; de Cisneros, B. J. Appl. Phys. A: Mater. Sci. Process. 2002, 75, 179.
(32) Sekimoto, K. J. Phvs. Soc. Jpn. 1997, 66, 1234.

(33) (a) Derényi, I.; Bier, M.; Astumian, R. D. Phvs. Rev. Lett. 1999, 83, 903. (b) Wang, H.; Oster, G. Europhys. Lett. 2002, 57, 134. (c) Suzuki, D.; Munakata, T. Phvs. Rev. E 2003, 68, 021906.

(34) Schmiedl, T.; Seifert, U. Europhys. Lett. 2008, 83, 30005.

(35) Schreier, M.; Reimann, P.; Hänggi, P.; Pollak, E. Europhvs. Lett. 1998, $44,416$.

(36) (a) Machura, L.; Kostur, M.; Talkner, P.; Łuczka, J.; Marchesoni, F.; Hänggi, P. Phys. Rev. E 2004, 70, 061105. (b) Linke, H.; Downton, M. T.; Zuckermann, M. J. Chaos 2005, 15, 026111. (c) Rozenbaum, V. M.; Korochkova, T. Ye.; Liang, K. K. Phys. Rev. E 2007, 75, 061115.

(37) Rozenbaum, V. M.; Korochkova, T. Ye.; Yang, D.-Y.; Lin, S. H.; Tsong, T. Y. Phvs. Rev. E 2005, 71, 041102.

(38) Talkner, P.; Łuczka, J. Phvs. Rev. E 2004, 69, 046109.

(39) Rozenbaum, V. M.; Yang, D.-Y.; Lin, S. H.; Tsong, T. Y. Physica A 2006, 363, 211

JP910508T 